\title{
Extending Phase Noise Tolerance in UDWDM Access Networks
}

\author{
Hubert Dzieciol \\ Optical Networks Group (ONG) \\ University College London \\ London, United Kingdom \\ hubert.dzieciol.18@ucl.ac.uk
}

\author{
Eric Sillekens \\ Optical Networks Group (ONG) \\ University College London \\ London, United Kingdom \\ e.sillekens@ucl.ac.uk
}

\author{
Domaniç Lavery \\ Optical Networks Group (ONG) \\ University College London \\ London, United Kingdom \\ d.lavery@ucl.ac.uk
}

\begin{abstract}
Geometrically shaped constellations, designed for improved AWGN channel performance, are combined with enhanced symbol decision metrics for low symbol rate/high linewidth applications, e.g., ultra-dense wavelength division multiplexed passive optical networks (UDWDM-PON). The improved metric enables 32-ary constellation transmission with robustness to AWGN and phase noise.

Index Terms-Ultra-dense wavelength division multiplexing, modulation formats, coherent optical communications
\end{abstract}

\section{INTRODUCTION}

Increasing bandwidth requirements in access networks have attracted a significant research interest in improved data rates in the of next-generation passive optical networks. A promising technology to deliver 50 and $100 \mathrm{~Gb} / \mathrm{s}$ links [1] are the simplified coherent schemes. Compared to intensity-modulated direct detection (IM-DD), coherent reception offers inherent wavelength selectivity by using a tunable local oscillator (LO) laser to recover the signal, as well as an increased receiver sensitivity.

The ultra-dense wavelength-multiplexing (UDWDM) scheme in access networks with 16-ary quadrature amplitude modulation (QAM) was first experimentally demonstrated in [2]. By operating at low symbol rates and dedicating a separate wavelength per optical network unit (ONU), it offers a much greater bandwidth utilisation and flexibility. However, it is often assumed that coherent detection at such low symbol rates would require a very low laser linewidth, e.g., $<100 \mathrm{kHz}$. Conversely, the majority of low-cost, monolithically integrated tunable lasers typically exhibit relatively high linewidths and therefore seem unsuitable for UDWDM-PONs.

In [3], we demonstrated an improvement in power budget when the modulation format was shaped for improved noise tolerance in an AWGN channel. However, this work assumed a high symbol rate, low linewidth transmission system, which would not be the case for a UDWDM-PON.

Therefore, this paper investigates techniques to improve the performance of a low symbol rate coherent UDWDM-PONs by jointly considering FEC and the modulation, i.e., coded modulation (CM). Moreover, we discuss the possible implementations of a pilot-aided carrier phase estimator (CPE), and identify the regimes where different $\mathrm{CM}$ strategies could be
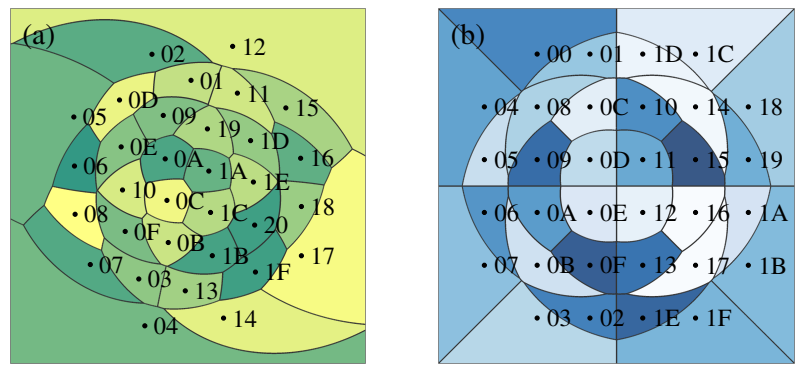

Fig. 1. The two-dimensional: (a) AWGN shaped 32-ary (GS32); (b) 32QAM; constellations at the indicative GMI threshold and a phase noise variance of a $1 \mathrm{MHz}$ lasers. The separated and distinctively coloured regions are the decision boundaries of the enhanced RPE used to further remove the residual phase noise (RPN) impairments. The binary bit-to-symbol mapping is presented as hexadecimal numbers.

used. Ultimately, we present a theoretical approach to relax laser linewidth requirements by applying an enhanced harddecision slicer to remove the residual phase noise (RPN) from the recovered signal and still demonstrate a power budget advantage over a comparable IM-DD transmission scheme.

\section{SYSTEM MODEL}

When dealing with UDWDM-PON transmission at low symbol rates and relatively high linewidth lasers, the memoryless optical fibre transmission is impaired by both AWGN and phase noise. Therefore, the channel can be modelled as $Y=X \cdot e^{j \phi_{n}}+\mathcal{N}$, where $X$ represents the transmitted symbol, $\mathcal{N}$ is a zero-mean Gaussian random variable, and $Y$ is the received symbol. Moreover, $\phi_{n}$ is also a Gaussian distributed random variable with variance $\sigma_{\mathrm{PN}}^{2}=2 \pi \Delta \nu \Delta T_{S}$, where $\Delta \nu$ is the sum of the signal and LO laser linewidths and $\Delta T_{S}$ is the symbol duration.

The channel was simulated using $2^{20}$ symbols per MonteCarlo channel realisation, including both AWGN and phase noise impairments. The symbol rate was 2.5 Gbaud. To put the results in context of next-generation PONs, we selected 32-ary modulation as being able to deliver net rates above 10 and $20 \mathrm{~Gb} / \mathrm{s}$ throughput using EPON-compatible FEC (single or dual polarisation transmission, respectively), as proposed in [3]. Herein, we paired the presented modulation formats with a 0.826 -rate binary soft-decision low-density parity check (LDPC) code, as per the 50/100G EPON standard [1]. Then, using a generalised mutual information (GMI) metric, which has been shown to be a reliable performance predictor for this 
LDPC scheme [3], we verified the performance of our system against the GMI threshold of $m \times 0.875 \mathrm{bit} / \mathrm{sym}$ using the same 32QAM and AWGN optimised (GS32) formats.

To keep carrier phase recovery format agnostic, pilot-aided carrier phase estimation (CPE) was employed [4]. The QPSK pilots were inserted between every 32 symbols of data. The resulting phase estimates were interpolated over the entire frame length using a Wiener filter with a minimum length of 65 taps, ensuring CPE across at least two pilot symbols. In practice, this CPE could then be enhanced using blind carrier phase recovery between the pilots. By subsequently assuming that the portion of the phase noise remaining after the pilotaided CPE is residual, and therefore has no random walk component, we can apply a residual phase estimator (RPE) to improve the overall CPE performance. The RPE makes an estimate of the residual phase noise by first removing the modulation by making a maximum likelihood symbol decision (minimum Euclidean distance) to produce a field estimate, and then taking the angle of the average field estimate over a 31sample sliding window.

Herein we also use the PCAWGN model in [5] to improve the accuracy of symbol estimation by improving the symbol decision regions (accounting for both phase noise and Gaussian noise), as originally proposed in [6]. This consequently improves the performance of the blind residual phase estimation algorithm [7]. An example of the improved decision regions is shown in Fig. 1(a) and (b).

\section{RESULTS}

We simulated the presented system using the shot noise model of a coherent receiver [8], assuming a receiver quantum efficiency of 0.1 . We analysed three transmission scenarios per constellation: with the enhanced RPE (solid lines), with the more typical Euclidean distance based RPE (dotted lines) and no RPE compensation (dashed lines). As depicted in Fig. 2 , given a $1 \mathrm{MHz}$ combined laser linewidth and no RPE the approximated power budgets are -23.0 and $-23.6 \mathrm{dBm}$ for the AWGN optimised (GS32) and 32QAM formats. Adding the euclidean distance RPE reduced the required receiver sensitivities to -25.5 (GS32-RPE) and $-25.3 \mathrm{dBm}$ (32QAMRPE), respectively. Moreover, by applying enhanced RPE, we were able to further mitigate these penalties and achieve the power budgets of $-26.5 \mathrm{dBm}\left(\mathrm{GS} 32-\mathrm{RPE}^{\star}\right)$ and $-26.0 \mathrm{dBm}$ (32QAM-RPE ${ }^{\star}$ ). Furthermore, under the assumption of using one of the two given compensation methods, the demonstrated approach allowed to reach the indicative GMI threshold at 1.5 MHz combined linewidth. Nevertheless, the SNR penalties are above $6 \mathrm{~dB}$ which, for Euclidean RPE, translates into the power budgets of -23.1 and $-23.4 \mathrm{dBm}$ for GS32 and 32QAM, respectively. However, employing the enhanced RPE model reduces them to $-25.0 \mathrm{dBm}$ for GS32 and $-24.3 \mathrm{dBm}$ for 32QAM.

\section{CONCLUSION}

The proposed CPE algorithm demonstrated the feasibility of transmitted 32-ary geometrically shaped formats at a

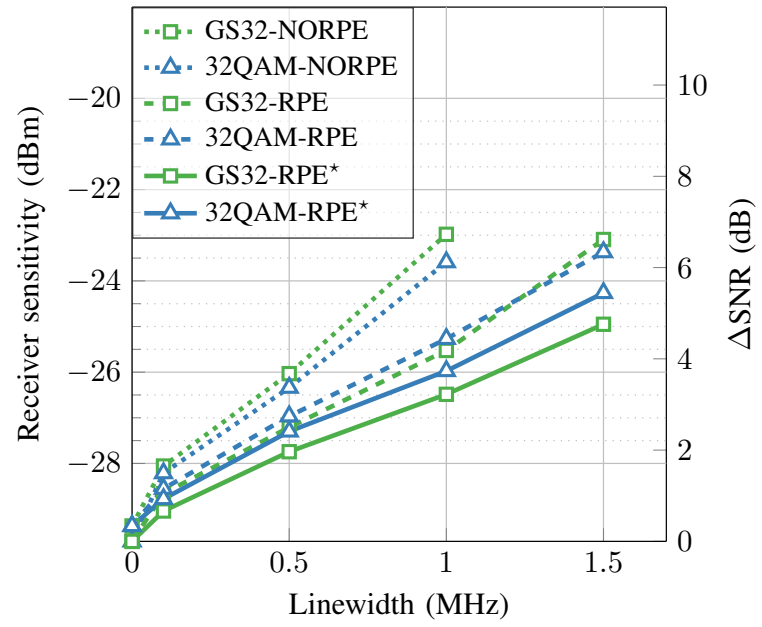

Fig. 2. SNR penalty vs combined laser linewidth at the indicative GMI threshold of $m \times 0.875 \mathrm{bit} / \mathrm{sym}$ for $2.5 \mathrm{GBd} 32$-ary modulation. RPE* indicates decision boundaries calculated using the PCAWGN channel model.

2.5 Gbaud symbol rate using a $1 \mathrm{MHz}$ laser linewidth. This can be translated into an effective post-FEC data rate of $10.325 \mathrm{Gbit} / \mathrm{s} /$ polarization (which could carry, for example, $10 \mathrm{G}$ Ethernet traffic). We observe that GS constellations, combined with an enhanced RPE model, can be used as a tool to substantially relax the laser linewidth requirements, therefore reducing the potential cost of implementing a UDWDM system. In our future work, we will investigate the impact of constellation shaping specifically for the PCAWGN channel on laser linewidth requirements.

\section{ACKNOWLEDGMENTS}

This work is part of the TRANSNET programme grant $(E P / R 035342 / 1)$. H. Dzieciol is in receipt of Ph.D. studentship from the EPSRC and Microsoft Research. The authors extend their thanks to Dr Martí S. Llopis for enlightening discussions.

\section{REFERENCES}

[1] "IEEE P802.3ca 50G-EPON Task Force." [Online]. Available: http://www.ieee802.org/3/ca/

[2] J. D. Reis, A. Shahpari, R. Ferreira, S. Ziaie, D. M. Neves, M. Lima, and A. L. Teixeira, "Terabit $(192 \times 10 \mathrm{~Gb} / \mathrm{s})$ Nyquist Shaped UDWDM Coherent PON With Upstream and Downstream Over a $12.8 \mathrm{~nm}$ Band," Journal of Lightwave Technology, vol. 32, no. 4, p. 729-735, 2014.

[3] T. Gerard, H. Dzieciol, E. Sillekens, Y. Wakayama, A. Alvarado, R. I. Killey, P. Bayvel, and D. Lavery, "Coded Modulation for 100G Coherent EPON," Journal of Lightwave Technology, vol. 38, no. 3, p. 564-572, Jan 2020

[4] M. Mazur, J. Schröder, A. Lorences-Riesgo, T. Yoshida, M. Karlsson, and P. A. Andrekson, "Overhead-optimization of pilot-based digital signal processing for flexible high spectral efficiency transmission," Opt. Express, vol. 27, no. 17, pp. 24654-24669, Aug 2019. [Online]. Available: http://www.opticsexpress.org/abstract.cfm?URI=oe$27-17-24654$

[5] M. Sales-Llopis and S. J. Savory, "Approximating the Partially Coherent Additive White Gaussian Noise Channel in Polar Coordinates," IEEE Photonics Technology Letters, vol. 31, no. 11, pp. 833-836, June 2019.

[6] M. Sales Llopis, "Optical fibre communication over a noisy partially coherent channel," Ph.D. dissertation, University of Cambridge, 2020.

[7] E. Ip and J. M. Kahn, "Feedforward carrier recovery for coherent optical communications," Journal of Lightwave Technology, vol. 25, no. 9, pp. 2675-2692, Sep. 2007 
[8] K. Kikuchi and S. Tsukamoto, "Evaluation of Sensitivity of the Digital Coherent Receiver," Journal of Lightwave Technology, vol. 26, no. 13, pp. 1817-1822, 2008. 